\title{
On a Darcy-Stefan Problem arising in freezing and thawing of saturated porous media*
}

\author{
José Francisco Rodrigues \\ C.M.A.F. / Universidade de Lisboa \\ Av. Prof. Gama Pinto, 2 - 1699 Lisboa Codex, Portugal \\ José Miguel Urbano \\ C.M.U.C. / Universidade de Coimbra \\ Apartado 3008 - 3000 Coimbra, Portugal
}

\begin{abstract}
A model with phase change for material convection in a saturated porous medium with a frozen region is formulated as a Darcy-Stefan problem. We propose a new generalized formulation for this Stefan-type problem with convection governed by Darcy's law. This approach, which is valid for irregular geometries with irregular subregions, has the advantage of not requiring the smoothness of the temperature, that restricted previous mathematical works to two-dimensional particular cases. We show existence of generalized solutions, passing to the limit in suitable approximated problems, which in principle can be solved numerically by the finite element method.
\end{abstract}

\section{INTRODUCTION}

In many problems in geodynamics and soil engineering the water-ice phase change plays an important role on natural convection in regions occupied by saturated porous media. The presence and evolution of the corresponding interface, as shown by experimental observations and theoretical analysis, have significant influence in the heat transfer and, consequently, also in the fluid motions. Because of the technical importance of this phenomenon, several models have been considered for concrete problems like in the construction of pipelines in permafrost regions, e.g. Goldstein and Reid [12], liquified gas or road construction and maintenance, e.g. Aguirre-Puente and Frémond [1], and so on.

"to appear in "Continuum Mechanics and Thermodynamics" 
More recently the melting of ice with natural convection in a porous medium has been considered in particular cases, e.g. Beckermann and Viskanta [4], Kazmierczak and Poulikakos [13] or Zhang et al. [24]. An interesting aspect of the natural convection with phase change in porous media is the analysis of patterned ground formation, e.g. George et al. [11]. Applying a linear instability analysis to a model of this geophysical phenomenon forming regular patterns, McKay [15] has analysed the effect of variable thermal parameters on fluid motions in the melted region.

Although phase change problems in porous media have specific features, they can be considered, at least from a theoretical point of view, as belonging to the large class of Stefan problems, since they are mathematically similar to the solidification of a metal piece or a semiconductor crystal, for which there exists an extensive literature (see e.g. Rubinstein [21], Meirmanov [16] or Rodrigues [18] and their bibliographies). When convection in the liquid phase is governed by Stokes or generalized Navier-Stokes equations, the corresponding mathematical analysis for evolutionary or steady-state cases has been considered in particular by DiBenedetto and O'Leary [10] or Rodrigues and Urbano [20], respectively (see also their bibliographies). Previous numerical computations based in the finite element modeling of a two-phase Rayleigh-Bénard problem can be found in Chang and Brown [6].

For a problem in ground freezing, the mathematical analysis of the model considered in Goldstein and Reid [12] was developed by DiBenedetto and Elliott [8] and by DiBenedetto and Friedman [9]. Their approach is restricted to the stationary two dimensional cases only, since it depends in a crucial manner on a regularity result for the temperature field, which is required to be continuous in order to give a sense to the flow region. In order to overcome this difficulty, Rodrigues [19] has proposed a new suitable generalized formulation for the case when there is no flow across the interface, which yields an interesting well posed mathematical problem, and proved a general existence theorem and, for small Péclet numbers, also a uniqueness result for the time-independent problem.

In this work we extend the new mathematical formulation for the evolutionary convection-conduction problem arising in freezing and thawing of saturated porous media. It consists of a two-phase Stefan problem with convection only in the liquid zone. The velocity field in the unfrozen domain is related to the hydraulic potential, or to the pressure, by Darcy's law with a buoyancy force that may depend on the temperature in a general form. For each instant, this yields a Neumann problem only in the liquid region, which is extended to the whole domain for mathematical convenience. Due to the solenoidal property of the velocity field, this extension is shown to be the natural one for the Neumann boundary condition across the free boundary, as it can be reobtained in the case of regular solutions from the generalized formulation, as shown in the next section.

Using a suitable regularization procedure, we give in section 3 quite general assumptions under which a priori estimates for the so obtained approximated 
problem garantee the existence of a generalized solution. Letting the approximating parameter go to zero we construct a subsequence of approximating solutions of the initial phase change problem with convection. This suggests in particular a numerical algorithm for computing actual solutions, which can be done by the finite element method, as for instance in the work of Barret and Elliott [2].

Although we do not consider here the numerical computation of the solution, the preliminary analysis of our section 4 , in particular the a priori estimates for the continuum approximated problem, can also be extended to the discrete problem of the standard Galerkin finite element approximation. It is perhaps worthwhile noting that our generalized approach is well adapted to the methodology of finite element simulations currently used in science and engineering (see e.g. Carey [5]) and allow the application of contemporary methods of optimal control of distributed systems (see e.g. Neittaanmäki and Tiba [17] and its bibliography).

\section{The mathematical Formulation}

We assume the porous medium occupies a bounded regular domain $\Omega \subset \mathbb{R}^{N}$, $(N=2,3)$, which is separated in two sets $\mathcal{S}$ and $\mathcal{L}$, corresponding respectively to the frozen and melted regions, which are divided by an unknown surface, the free boundary $\Phi$.

According to the continuum approach to porous media (see, for instance, Bear [3]), we consider the equation of the energy

$$
\frac{\partial e}{\partial t}+\mathbf{v} \cdot \nabla e=r-\nabla \cdot \mathbf{q}
$$

where we assume a normalized constant density $\rho \equiv 1$ and denote by $\mathbf{q}$ the energy flux, $r$ the energy source, $e$ the internal energy and $\mathbf{v}$ the velocity field. The classical Fourier law is taken as constitutive relation between the temperature field and the heat flux and it reads

$$
\mathbf{q}=-k(T) \nabla T
$$

where $k$ denotes the thermal conductivity. One of the assumptions in the classical Stefan problem is that the change of phase occurs through $\Phi$ at a fixed temperature $T_{\Phi}$ and introducing the usual Kirchhoff transformation, we define the normalized temperature by

$$
\theta \equiv K(T)=\int_{T_{\Phi}}^{T} k(\tau) \mathrm{d} \tau
$$

and relation (1) becomes simply $\mathbf{q}=-\nabla \theta$. We can then write, respectively, for the frozen and unfrozen zones,

$$
\mathcal{S}=\{(x, t) \in Q: \theta(x, t)<0\} \quad, \quad \mathcal{L}=\{(x, t) \in Q: \theta(x, t)>0\}
$$




$$
\text { and } \Phi=\{(x, t) \in Q: \theta(x, t)=0\},
$$

for the unknown interface. We denote the given space-time domain by $Q=$ $\Omega \times(0, T)$, for some $T>0$, and its lateral boundary by $\Sigma=\partial \Omega \times(0, T)$.

The constitutive relation between the internal energy and the temperature field takes into account the jump that occurs at the interface, and we assume

$$
e(T)=e\left(K^{-1}(\theta)\right)=d(\theta)+\operatorname{lh}(\theta)
$$

where $d$ is a given continuous and strictly increasing function, $l=[e]_{-}^{+}>0$ is the latent heat of phase transition, with $[.]_{-}^{+}$denoting the jump across $\Phi$, and $h$ is the Heaviside function $(h(s)=0$ if $s<0, h(s)=1$ if $s>0)$. Without loss of generality, we may assume that $d(0)=0$.

From the energy conservation law and using the two constitutive relations, with $r \equiv 0$ for simplicity, we obtain the heat diffusion equation with convection

$$
\partial_{t} d(\theta)+\mathbf{v} \cdot \nabla d(\theta)=\Delta \theta \quad \text { in } \quad Q \backslash \Phi=\{\theta<0\} \cup\{\theta>0\} .
$$

On the free boundary $\Phi$, in addition to the condition $\theta=0$, we have the Stefan condition (see, for instance, Rodrigues [18]), which represents the balance of heat fluxes

$$
[\mathbf{q}]_{-}^{+} \cdot \mathbf{n}=[-\nabla \theta]_{-}^{+} \cdot \mathbf{n}=l(\mathbf{v}-\mathbf{w}) \cdot \mathbf{n} \quad \text { on } \quad \Phi=\{\theta=0\} .
$$

Here, $\mathbf{n}$ is the unit normal to $\Phi$, pointing to the solid region and $\mathbf{w}$ is the velocity of the free boundary.

The boundary and initial conditions are, respectively

$$
\begin{gathered}
-\frac{\partial \theta}{\partial \mathbf{n}}=f(x, t, \theta) \quad \text { on } \quad \Sigma ; \\
\theta(0)=\theta_{0} \quad \text { in } \quad \Omega,
\end{gathered}
$$

for a given initial data $\theta_{0}$ and a given, possibly nonlinear, heat flux $f$.

Concerning the velocity field $\mathbf{v}$, by the Boussinesq approximation we assume it satisfies in the melted zone the continuity equation and Darcy's law, i.e.,

$$
\left.\begin{array}{l}
\nabla \cdot \mathbf{v}=0 \\
\mathbf{v}=-\nabla p+\mathbf{b}(\theta)
\end{array}\right\} \text { in }\{\theta>0\}
$$

$p$ being the dimensionless hydraulic potential and $\mathbf{b}=\mathbf{b}(\theta)$ a buoyance force which can be a nonlinear function of the temperature (see, e.g., Straughan [23] or McKay [15] and their bibliographies). On the frozen zone, we have

$$
\mathbf{v}=0 \quad \text { in } \quad\{\theta<0\},
$$


and the interface boundary condition is

$$
\mathbf{v} \cdot \mathbf{n}=0 \quad \text { on } \quad \partial\{\theta>0\}
$$

with $\mathbf{n}$ denoting the exterior normal vector to $\partial\{\theta>0\}$.

We see that the hydraulic potential solves the Neumann problem

$$
\Delta p=\nabla \cdot \mathbf{b}(\theta) \quad \text { in } \quad\{\theta>0\} \quad, \quad \partial p / \partial \mathbf{n}=\mathbf{b}(\theta) \cdot \mathbf{n} \quad \text { on } \quad \partial\{\theta>0\}
$$

in the a priori unknown domain $\{\theta>0\}$. This is a delicate problem since it requires the temperature to be at least a lower semicontinuous function so that $\{\theta>0\}$ is open and the Poisson equation (9) makes sense in a variational form. This is exactly the approach of the weak formulation introduced in DiBenedetto and Elliott [8] in a steady state situation, where the temperature is required to be a continuous function. The boundary condition $\partial p / \partial \mathbf{n}=\mathbf{b}(\theta) \cdot \mathbf{n}$ on $\partial\{\theta>0\} \cap$ $\partial \Omega$ is not taken into account in the problem formulation and the mathematical results obtained only hold in the particular two-dimensional setting.

We now derive a generalized formulation for the problem. In what concerns the Stefan problem we consider the now classical enthalpy formulation (cf. Meirmanov [16] and the references therein). Let $H$ denote the maximal monotone graph associated with the Heaviside function,

$$
H(s)=\left\{\begin{array}{ccc}
0 & \text { if } & s<0 \\
{[0,1]} & \text { if } & s=0 \\
1 & \text { if } & s>0
\end{array},\right.
$$

and define the dimensionless enthalpy by the pointwise inclusion

$$
\eta \in \gamma(\theta)=d(\theta)+l H(\theta)
$$

Integrating formally by parts equation (2), with a smooth test function $\xi$ such that $\xi(T)=0$, assuming that $\Phi$ is smooth and taking into account the jump of $\gamma$ at 0 and the boundary conditions, in particular, conditions (3) and (8) (see, for instance, Rodrigues [18]), we get

$$
-\int_{Q} \eta \partial_{t} \xi-\int_{Q} d(\theta) \mathbf{v} \cdot \nabla \xi+\int_{Q} \nabla \theta \cdot \nabla \xi+\int_{\Sigma} f(\theta) \xi=\int_{\Omega} \eta_{0} \xi(0),
$$

where the initial condition on the enthalpy, which carries more information than the corresponding one on the temperature, is chosen such that $\eta_{0} \in \gamma\left(\theta_{0}\right)$. We observe that the convective term depends only on the temperature and not on the enthalpy, which is due to the interface condition (8).

As far as the velocity is concerned, the generalized formulation that we propose consists in solving the Neumann problem (9) in the whole domain $\Omega$, instead of only in $\{\theta>0\}$ and defining $\mathbf{v}$ a posteriori by the expression

$$
\mathbf{v}=[-\nabla p+\mathbf{b}(\theta)] \chi_{\{\theta \geq 0\}},
$$


where $\chi_{A}$ denotes the characteristic function of a set $A$. This is consistent with (6) and (7) and based on the simple remark that

$$
d(\theta) \mathbf{v}=d^{+}(\theta)[-\nabla p+\mathbf{b}(\theta)]=d(\theta)[-\nabla p+\mathbf{b}(\theta)] \chi_{\{\theta \geq 0\}},
$$

with

$$
d^{+}(\theta)=\max (d(\theta), 0)=\left\{\begin{array}{cl}
d(\theta) & \text { if } \quad \theta \geq 0 \\
0 & \text { if } \quad \theta \leq 0
\end{array} .\right.
$$

We comment later on how to recover the interface condition (8).

Concerning notation, we use the usual symbols for the function spaces involved, in particular, the usual Sobolev spaces $H^{1}(\Omega)$ and

$$
H^{1}(Q)=H^{1}\left(0, T ; L^{2}(\Omega)\right) \cap L^{2}\left(0, T ; H^{1}(\Omega)\right) .
$$

The definition of generalized solution is the following

Definition. We say that $(\theta, \eta, p)$ is a generalized solution of the Darcy-Stefan problem, if

$$
\begin{gathered}
\theta \in L^{2}\left(0, T ; H^{1}(\Omega)\right) \cap L^{\infty}(Q) ; \\
\eta \in L^{\infty}(Q) \text { and } \eta \in \gamma(\theta), \text { a.e. in } Q ; \\
p \in L^{\infty}\left(0, T ; H^{1}(\Omega) / \mathbb{R}\right) ; \\
-\int_{Q} \eta \partial_{t} \xi+\int_{Q} \nabla \theta \cdot \nabla \xi-\int_{Q} d^{+}(\theta)[-\nabla p+\mathbf{b}(\theta)] \cdot \nabla \xi+\int_{\Sigma} f(\theta) \xi \\
=\int_{\Omega} \eta_{0} \xi(0), \quad \forall \xi \in H^{1}(Q): \xi(T)=0 ; \\
\int_{\Omega}[-\nabla p+\mathbf{b}(\theta)] \cdot \nabla \psi=0, \quad \forall \psi \in H^{1}(\Omega), \text { a.e. } t \in(0, T] .
\end{gathered}
$$

Remark 1 We stress that the temperature is not required to be continuous, as in DiBenedetto and Elliott [8] and DiBenedetto and Friedman [9], at the cost of obtaining as a solution of the Neumann problem (16) an artificial hydraulic potential $p$ defined in the whole domain $\Omega$ for a.e. $t \in(0, T]$. The function $p(t)$ is defined up to a constant, which is traduced by the fact that it belongs to the quocient space $H^{1}(\Omega) / \mathbb{R}$. We stress that only the contribution of its gradient in the measurable subset $\{\theta>0\}$ is relevant to the conduction-convection problem (15) and that, with definition (11), we recover the velocity field. 
Remark 2 In the formulation of DiBenedetto and Elliott [8], the condition $\nabla \cdot \mathbf{v}=0$ was to hold globally and $p$ was required to belong only to $H^{1}(\{\theta>0\})$, since, due to the continuity of $\theta$, the set $\{\theta>0\}$ was open. The variational equation (16) was then restricted only to the $\psi \in H^{1}(\Omega)$ such that supp $\psi \subset$ $\{\theta>0\}$.

Remark 3 Here, equation (16), with definition (11), contains in a generalized sense the boundary condition (8), i.e., $p$ solves the Neumann problem (9) in a generalized sense. Indeed, as was already observed in Rodrigues [19], if, for a.e. $t$, the free boundary $\Gamma \equiv \Phi(t)=\{\theta(t)=0\} \cap \Omega(t)=\partial\{\theta(t)>0\} \cap \Omega(t)$ is regular (for instance, of class $C^{1}$ ) and $\nabla \theta$ and $-\nabla p+\mathbf{b}(\theta)$ are continuous in a neighbourhood of $\Gamma$, its normal vector, say $\mathbf{n}_{\Gamma}$, is parallel to $\left.\nabla \theta\right|_{\Gamma}$. Hence, for $\delta>0$ sufficiently small, defining

$$
H_{\delta}(t)=1 \text { if } t \geq \delta, \quad H_{\delta}(t)=t / \delta \text { if } 0 \leq t \leq \delta \text { and } H_{\delta}(t)=0 \text { if } t \leq 0 \text {, }
$$

and choosing $\psi=H_{\delta}(\varphi \theta)$ in (16), where $\varphi \in C^{1}(\bar{\Omega}), \varphi>0$ in $\bar{\Omega}$, is arbitrary, we obtain

$$
\int_{\{0<\varphi \theta<\delta\}}[-\nabla p+\mathbf{b}(\theta)] \cdot \nabla(\varphi \theta) \mathrm{d} x=0 .
$$

Dividing by meas $\{0<\varphi \theta<\delta\}$ and letting $\delta \rightarrow 0$, we find

$$
\int_{\{\theta=0\} \cap \Omega}[-\nabla p+\mathbf{b}(\theta)] \cdot \nabla(\varphi \theta) \mathrm{d} \Gamma=\int_{\Gamma} \varphi[-\nabla p+\mathbf{b}(\theta)] \cdot \nabla \theta \mathrm{d} \Gamma=0
$$

first for positive $\varphi$ and then also for any $\varphi \in C(\Gamma)$. We conclude that

$$
[-\nabla p+\mathbf{b}(\theta)] \cdot \mathbf{n}_{\Gamma}=0, \text { everywhere on } \Gamma .
$$

Remark 4 Instead of the Neumann condition (4) we may also consider a Dirichlet or a mixed Dirichlet-Neumann boundary condition, i.e., for a partition $\Sigma=\Sigma_{D} \cup \Sigma_{N}$,

$$
\theta=\theta_{D} \quad \text { on } \quad \Sigma_{D} \quad \text { and } \quad-\frac{\partial \theta}{\partial n}=f(\theta) \text { on } \Sigma_{N} .
$$

In particular, we can approach the Dirichlet condition by a family of Neumann type problems in the form

$$
f(x, t, \theta)=\sigma\left[\theta-\theta_{D}(x, t)\right], \text { for a.e. }(x, t) \in \Sigma_{D},
$$

by letting the parameter $\sigma \rightarrow+\infty$.

Remark 5 In most geophysical problems, the thermoconvection takes place in anisotropic and heterogeneous porous media. From the mathematical point of view, the corresponding laws (1) and (6) require the introduction of the additional conductivity and permeability tensor fields, and the generalized formulation (15)-(16) may be easily extended to this case, as well as the theoretical results of the next sections. 


\section{Existence of generalized solutions}

In this section we show that the problem admits at least one generalized solution in the sense of our definition. The proof consists in a regularization of the maximal monotone graph and some data, giving rise to an approximated problem, for which solvability is obtained using a fixed point theorem and standard results from parabolic theory. The a prioriestimates, that allow the consequent passage to the limit, are obtained in the next section. We state the assumptions and the theorem and then present its proof.

(A1) $d \in C^{0,1}(\mathbb{R})$ is such that $d(0)=0$ and $0<\underline{d} \leq d^{\prime}(s) \leq \bar{d}$, a.e. $s \in \mathbb{R}$;

(A2) $\mathbf{b} \in C^{0}\left(\mathbb{R} ; \mathbb{R}^{N}\right)$;

(A3) $\eta_{0} \in \gamma\left(\theta_{0}\right)$, with $\theta_{0} \in L^{\infty}(\Omega)$;

(A4) $f: \Sigma \times \mathbb{R} \rightarrow \mathbb{R}$ is a measurable function with $f(x, t, \cdot)$ increasing and continuous for a.e. $(x, t) \in \Sigma$;

(A5) There exists a constant $M>0$ such that

$$
\begin{aligned}
& \text { (i) } \quad|| \theta_{0} \|_{L^{\infty}(\Omega)} \leq M \\
& \text { (ii) } \quad f(x, t, \tau) \tau \geq 0 \text { for }|\tau| \geq M, \text { a.e. }(x, t) \in \Sigma \\
& \text { (iii) } \exists f^{*} \in L^{\infty}(\Sigma): \sup _{|\tau| \leq M}|f(x, t, \tau)| \leq f^{*}(x, t), \text { a.e. in } \Sigma
\end{aligned}
$$

Theorem 1 Under the previous assumptions, there exists at least one generalized solution to the Darcy-Stefan problem in the sense of (12)-(16), such that

$$
\|\theta\|_{L^{\infty}(Q)} \leq M
$$

Proof. We shall consider an approximated problem, that is solved in the next section, and obtain the solution as the limit of the approximated solutions. We start with a regularization of the maximal monotone graph $\gamma$. Let $0<\epsilon<1$ and consider the function

$$
\gamma_{\epsilon}(s)=d(s)+l H_{\epsilon}(s)
$$

where $H_{\epsilon}$ is a $\mathcal{C}^{\infty}$-approximation of the Heaviside function, such that

$$
H_{\epsilon}(s)=0 \quad \text { if } \quad s \leq 0, H_{\epsilon}(s)=1 \quad \text { if } \quad s \geq \epsilon \quad \text { and } \quad H_{\epsilon}^{\prime}(s) \geq 0, s \in \mathbb{R}
$$


with $H_{\epsilon} \rightarrow h$ uniformly in the compact subsets of $\mathbb{R} \backslash\{0\}$, as $\epsilon \rightarrow 0$. The function $\gamma_{\epsilon}$ is bilipschitz and satisfies

$$
0<\underline{d} \leq \gamma_{\epsilon}^{\prime}(s) \leq \overline{d_{\epsilon}}=\bar{d}+l L_{\epsilon}, \quad \text { a.e. } s \in \mathbb{R},
$$

with $L_{\epsilon} \equiv \mathcal{O}\left(\frac{1}{\epsilon}\right)$ being the Lipschitz constant of $H_{\epsilon}$. Its inverse $\beta_{\epsilon}=\gamma_{\epsilon}^{-1}$ satisfies

$$
0<\frac{1}{\overline{d_{\epsilon}}} \leq \beta_{\epsilon}^{\prime}(s) \leq \frac{1}{\underline{d}}, \text { a.e. } s \in \mathbb{R} .
$$

We also regularize the boundary data with respect to the time variable by considering functions $f_{\epsilon}^{M}(x, t, \tau)$, satisfying (A4) and (A5)-(ii)(iii), such that, for each $\epsilon>0$,

$$
\sup _{\tau}\left|\partial_{t} f_{\epsilon}^{M}\right| \in L^{2}(\Sigma) \quad \text { and } \quad \sup _{|\tau| \leq M}\left|f_{\epsilon}^{M}-f^{M}\right| \rightarrow 0 \quad \text { in } \quad L^{2}(\Sigma),
$$

denoting the truncation of a function $\phi$ with

$$
\phi^{M}(x, t, \tau)=\phi(x, t, \min \{M, \max (-M, \tau)\}) .
$$

Concerning the initial data, we consider a sequence of functions $\theta_{0 \epsilon} \in H^{1}(\Omega)$ such that, with $\eta_{0 \epsilon}=\gamma_{\epsilon}\left(\theta_{0 \epsilon}\right)$,

$$
\theta_{0 \epsilon} \rightarrow \theta_{0}, \quad \eta_{0 \epsilon} \rightarrow \eta_{0} \text { in } L^{2}(\Omega) \text { and }\left\|\theta_{0 \epsilon}\right\|_{L^{\infty}(\Omega)} \leq M
$$

The approximated problem is

$\left(\mathbf{P}_{\epsilon}\right)$ : For each $\epsilon>0$, find a pair $\left(\theta_{\epsilon}, p_{\epsilon}\right)$ such that

$$
\begin{array}{r}
\theta_{\epsilon} \in H^{1}(Q) \cap L^{\infty}(Q) \quad, \quad p_{\epsilon} \in L^{\infty}\left(0, T ; H^{1}(\Omega) / \mathbb{R}\right) ; \\
-\int_{Q} \gamma_{\epsilon}\left(\theta_{\epsilon}\right) \partial_{t} \xi+\int_{Q} \nabla \theta_{\epsilon} \cdot \nabla \xi-\int_{Q} d^{+}\left(\theta_{\epsilon}\right) \mathbf{w}_{\epsilon}^{*} \cdot \nabla \xi+\int_{\Sigma} f_{\epsilon}^{M}\left(\theta_{\epsilon}\right) \xi \\
=\int_{\Omega} \eta_{0 \epsilon} \xi(0), \quad \forall \xi \in H^{1}(Q): \xi(T)=0 ; \\
\int_{\Omega}\left[-\nabla p_{\epsilon}+\mathbf{b}\left(\theta_{\epsilon}\right)\right] \cdot \nabla \psi=0, \quad \forall \psi \in H^{1}(\Omega), \text { a.e. } t \in(0, T]
\end{array}
$$

Here, $\mathbf{w}_{\epsilon}^{*}$ is a regularization of $\mathbf{w}_{\epsilon}:=-\nabla p_{\epsilon}+\mathbf{b}\left(\theta_{\epsilon}\right)$ that preserves its solenoidal nature, for example the convolution $\mathbf{w}_{\epsilon}^{*}=\rho_{\epsilon} * \mathbf{w}_{\epsilon}$, with a mollifier $\rho_{\epsilon}$ $\left(\rho_{\epsilon} \in \mathcal{C}^{\infty}, \int \rho_{\epsilon}=1, \rho_{\epsilon}(s)=0,|s| \geq 1\right)$. 
In the next section we show that this problem has a solution for each $\epsilon>0$ and establish the a priori estimates (31)-(34). From these estimates we conclude that for subsequences, relabeled with the same index, we have

$$
\begin{aligned}
& \theta_{\epsilon} \longrightarrow \theta \quad \text { in } \quad L^{\infty}(Q) \text { weak-*, } \\
& \text { in } L^{2}\left(0, T ; H^{1}(\Omega) \text { weak },\right. \\
& \text { in } \quad L^{2}(Q) \text { strong; } \\
& \gamma_{\epsilon}\left(\theta_{\epsilon}\right) \rightarrow \eta \quad \text { in } \quad L^{\infty}(Q) \text { weak-*; } \\
& \nabla p_{\epsilon} \rightarrow \nabla p \quad \text { in } \quad L^{\infty}\left(0, T ; L^{2}(\Omega)\right) \text { weak-*; } \\
& \mathbf{w}_{\epsilon} \rightarrow \mathbf{w} \text { and } \quad \mathbf{w}_{\epsilon}^{*} \rightarrow \mathbf{w} \quad \text { in } \quad L^{\infty}\left(0, T ; L^{2}(\Omega)\right) \text { weak-* },
\end{aligned}
$$

for functions $\theta \in L^{2}\left(0, T ; H^{1}(\Omega)\right) \cap L^{\infty}(Q)$ and satisfying (17), $\eta \in L^{\infty}(Q)$, $p \in L^{\infty}\left(0, T ; H^{1}(\Omega) / \mathbb{R}\right)$ and with $\mathbf{w}=-\nabla p+\mathbf{b}(\theta)$. The strong convergence $\theta_{\epsilon} \rightarrow \theta$ in $L^{2}(Q)$ follows, for example, from Theorem 1 in Simon [22], using estimates (32) and (33) and it also implies that $\mathbf{w}_{\epsilon} \rightarrow \mathbf{w}$ also strongly in $L^{2}$ since

$$
\begin{gathered}
\left\|\nabla p_{\epsilon}-\nabla p\right\|_{L^{2}}^{2}=\int_{\Omega} \nabla p_{\epsilon} \cdot\left(\nabla p_{\epsilon}-\nabla p\right)-\int_{\Omega} \nabla p \cdot\left(\nabla p_{\epsilon}-\nabla p\right) \\
=-\int_{\Omega}\left[\mathbf{b}\left(\theta_{\epsilon}\right)-\mathbf{b}(\theta)\right] \cdot\left(\nabla p_{\epsilon}-\nabla p\right) \longrightarrow 0,
\end{gathered}
$$

using Lebesgue's theorem and (28).

With this convergences, passing to the limit in (24) and (25) is straightforward since the boundary term converges due to (21) and lemma 4.6. in Rodrigues [18]. It remains to show that $\eta \in \gamma(\theta)$; observe that $\gamma_{\epsilon}$ is increasing and $\gamma_{\epsilon}=\beta_{\epsilon}^{-1}$, which gives

$$
\int_{Q}\left[\gamma_{\epsilon}\left(\theta_{\epsilon}\right)-\varphi\right]\left[\theta_{\epsilon}-\beta_{\epsilon}(\varphi)\right] \geq 0, \forall \varphi \in L^{\infty}(Q),
$$

and passing to the limit and using standard monotonicity techniques (cf. Rodrigues [18], for example) we obtain $\theta=\beta(\eta) \Leftrightarrow \eta \in \gamma(\theta)$ and conclude that $(\theta, \eta, p)$ is a solution to the Darcy-Stefan problem in the sense of the definition above.

Remark 6 For the Dirichlet or mixed boundary value problems see Rodrigues [18, Theorem 4.14] where a continuous dependence result allows one to obtain the solution for these problems as the limit of solutions of Neumann problems in the class referred to in Remark 4. 


\section{Analysis of the approximated PRoblem}

We show here that, for each $\epsilon>0$, the approximated problem has a solution, making use of a fixed point theorem. We then obtain the a prioriestimates that enabled us to pass to the limit, obtaining the solution of our problem.

Proposition 1 For each $\epsilon>0$, the approximated problem has at least one solution.

Proof. We fix $\epsilon>0$ and apply Schauder's fixed point theorem to a non-linear operator $\mathcal{T}_{\epsilon}$ defined in the Hilbert space $L^{2}(Q)$. For each $\sigma \in L^{2}(Q)$ such that $\|\sigma\|_{L^{\infty}(Q)} \leq M$, and using elementary methods for elliptic equations, we solve up to constants the linear Neumann problem (25), with $\theta_{\epsilon}=\sigma$, obtaining a unique $p_{\epsilon}^{\sigma} \in L^{\infty}\left(0, T ; H^{1}(\Omega) / \mathbb{R}\right)$. Next, we define $\mathbf{u}^{\sigma}=\left[-\nabla p_{\epsilon}^{\sigma}+\mathbf{b}(\sigma)\right]$ and solve the problem (24), with $\mathbf{w}_{\epsilon}^{*}$ replaced by $\mathbf{u}^{*}=\rho_{\epsilon} * \mathbf{u}^{\sigma}$, using results from parabolic theory. This problem can be written as

$$
\left\{\begin{array}{ll}
\partial_{t} \eta-\Delta \gamma_{\epsilon}^{-1}(\eta)+\mathbf{u}^{*} \cdot \nabla d^{+}\left(\gamma_{\epsilon}^{-1}(\eta)\right)=0 & \text { in } Q \\
\nabla \gamma_{\epsilon}^{-1}(\eta) \cdot \mathbf{n}+f_{\epsilon}^{M}\left(\gamma_{\epsilon}^{-1}(\eta)\right)=0 & \text { on } \Sigma \\
\eta(0)=\eta_{0 \epsilon} & \text { on } \Omega
\end{array} .\right.
$$

For the existence, we can use the Galerkin method as in Rodrigues [18], observing that the diferences with respect to the problem considered there are not significant since $\mathbf{u}^{*}$ is a solenoidal $L^{\infty}$ function and $g \equiv d^{+} \circ \gamma_{\epsilon}^{-1}$ is Lipschitz. The uniqueness, with the assumption on the monotonicity of the flux $f_{\epsilon}^{M}$, follows from a simple generalization of the results of Chipot and Rodrigues [7] to the Neumann case.

We obtain a function $\eta \in H^{1}(Q) \cap L^{\infty}(Q)$ and put $\gamma_{\epsilon}^{-1}(\eta):=\mathcal{T}_{\epsilon}(\sigma)$, that belongs to the same spaces and satisfies the estimates

$$
\left\|\mathcal{T}_{\epsilon}(\sigma)\right\|_{L^{\infty}(Q)} \leq M \quad \text { and } \quad\left\|\mathcal{T}_{\epsilon}(\sigma)\right\|_{H^{1}(Q)} \leq C_{\epsilon, M}
$$

respectively by the weak maximum principle (see Rodrigues [18, Prop. 4.7.]) and standard parabolic estimates. Therefore, $\mathcal{T}_{\epsilon}$ maps the closed and convex subset of $L^{2}(Q)$

$$
S_{M}=\left\{\sigma \in L^{2}(Q):\|\sigma\|_{L^{\infty}(Q)} \leq M\right\}
$$

into itself and is completely continuous, due to standard continuous dependence results of elliptic and parabolic theory and the compactness of the injection $H^{1}(Q) \hookrightarrow L^{2}(Q)$. So it has a fixed point $\theta_{\epsilon}=\mathcal{T}_{\epsilon}\left(\theta_{\epsilon}\right)$ and we obtain that $\left(\theta_{\epsilon}, p_{\epsilon}^{\theta_{\epsilon}}\right)$ solves the problem $\left(\mathbf{P}_{\epsilon}\right)$. 
Remark 7 Concerning the continuous dependence, we observe that if $\sigma_{\delta} \rightarrow \sigma_{0}$ strongly in $L^{2}$ then $\mathbf{w}_{\delta} \rightarrow \mathbf{w}_{0}$ also strongly in $L^{2}$, which can be achieved with a reasoning similar to the one that led to $(30)$.

Proposition 2 Any solution of the approximated problem satisfy the following independent of $\epsilon$ estimates:

$$
\begin{gathered}
\left\|\theta_{\epsilon}\right\|_{L^{\infty}(Q)} \leq M,\left\|\gamma_{\epsilon}\left(\theta_{\epsilon}\right)\right\|_{L^{\infty}(Q)} \leq M^{\prime} ; \\
\left\|\nabla \theta_{\epsilon}\right\|_{L^{2}(Q)} \leq C ; \\
\int_{0}^{T-h} h^{-1}\left\|\theta_{\epsilon}(t+h)-\theta_{\epsilon}(t)\right\|_{L^{2}(\Omega)}^{2} \mathrm{~d} t \leq \kappa, \quad \forall 0<h<T ; \\
\sup _{0<t<T}\left\|\nabla p_{\epsilon}(t)\right\|_{L^{2}(\Omega)} \leq C ;
\end{gathered}
$$

Proof. Since also $\gamma_{\epsilon}\left(\theta_{\epsilon}\right) \in H^{1}\left(0, T ; L^{2}(\Omega)\right) \subset C\left([0, T] ; L^{2}(\Omega)\right)$, we can integrate (24) by parts, and, after a careful choice for the test functions, conclude that $\gamma_{\epsilon}\left(\theta_{\epsilon}\right)(0)=\eta_{0 \epsilon}$ and that, for a.e. $t \in(0, T)$,

$$
\begin{aligned}
\int_{\Omega} \partial_{t} \gamma_{\epsilon}\left(\theta_{\epsilon}\right) \varphi+\int_{\Omega} \nabla \theta_{\epsilon} \cdot \nabla \varphi & -\int_{\Omega} d^{+}\left(\theta_{\epsilon}\right) \mathbf{w}_{\epsilon}^{*} \cdot \nabla \varphi \\
& +\int_{\partial \Omega} f_{\epsilon}^{M}\left(\theta_{\epsilon}\right) \varphi=0, \quad \forall \varphi \in H^{1}(\Omega) .
\end{aligned}
$$

To get the $L^{\infty}$-bound, take $\varphi=\left(\theta_{\epsilon}(\tau)-M\right)^{+} \in H^{1}(\Omega)$ in (35) and defining

$$
\tilde{\beta}_{M}(s)=\left\{\begin{array}{clc}
\int_{0}^{s}\left(\beta_{\epsilon}(\sigma)-M\right)^{+} \mathrm{d} \sigma & \text { if } & s>\gamma_{\epsilon}(M) \\
0 & \text { if } & s \leq \gamma_{\epsilon}(M)
\end{array},\right.
$$

obtain, after integration in time in $(0, t)$ and denoting with $\mu$ a function such that $\mu^{\prime}=d^{+}$,

$$
\begin{gathered}
\int_{\Omega} \tilde{\beta}_{M}\left(\gamma_{\epsilon}\left(\theta_{\epsilon}(t)\right)\right)+\int_{0}^{t} \int_{\Omega}\left|\nabla\left(\theta_{\epsilon}-M\right)^{+}\right|^{2} \\
=\int_{\Omega} \tilde{\beta}_{M}\left(\gamma_{\epsilon}\left(\theta_{\epsilon}(0)\right)\right)+\int_{0}^{t} \int_{\Omega} \mathbf{w}_{\epsilon}^{*} \cdot \nabla \mu\left(\left(\theta_{\epsilon}-M\right)^{+}+M\right) \\
-\int_{0}^{t} \int_{\partial \Omega} f_{\epsilon}^{M}\left(\theta_{\epsilon}\right)\left(\theta_{\epsilon}-M\right)^{+} \leq 0,
\end{gathered}
$$


because $\mathbf{w}_{\epsilon}^{*}$ is solenoidal and (A5)-(ii) holds for $f_{\epsilon}^{M}$. Since $t>0$ is arbitrary, we conclude

$$
\left(\theta_{\epsilon}-M\right)^{+}=0 \text {, i.e. } \theta_{\epsilon} \leq M \text { a.e. in } Q
$$

Analogously, we would get $\theta_{\epsilon} \geq-M$, by taking $\varphi=\left(\theta_{\epsilon}(\tau)+M\right)^{-}$in (35). Now, this estimate easily gives

$$
\left\|\gamma_{\epsilon}\left(\theta_{\epsilon}\right)\right\|_{L^{\infty}(Q)}=\left\|d\left(\theta_{\epsilon}\right)+l H_{\epsilon}\left(\theta_{\epsilon}\right)\right\|_{L^{\infty}(Q)} \leq \max _{|s| \leq M}|d(s)|+l \equiv M^{\prime}
$$

To get $(32)$, choose $\varphi=\theta_{\epsilon}(t)$ in $(35)$ and integrate in time, obtaining

$$
\int_{0}^{T} \int_{\Omega} \partial_{t} \hat{\gamma}_{\epsilon}\left(\theta_{\epsilon}\right)+\int_{Q}\left|\nabla \theta_{\epsilon}\right|^{2}-\int_{Q} \mathbf{w}_{\epsilon}^{*} \cdot \nabla \mu\left(\theta_{\epsilon}\right)+\int_{\Sigma} f_{\epsilon}^{M}\left(\theta_{\epsilon}\right) \theta_{\epsilon}=0,
$$

where

$$
\widehat{\gamma}_{\epsilon}(s)=\int_{0}^{\gamma_{\epsilon}(s)} \beta_{\epsilon}(\tau) \mathrm{d} \tau
$$

So we have, using again the fact that $\mathbf{w}_{\epsilon}^{*}$ is solenoidal,

$$
\begin{aligned}
\int_{Q}\left|\nabla \theta_{\epsilon}\right|^{2} & =\int_{\Omega} \hat{\gamma}_{\epsilon}\left(\theta_{\epsilon}(0)\right)-\int_{\Omega} \hat{\gamma}_{\epsilon}\left(\theta_{\epsilon}(T)\right)-\int_{\Sigma} f_{\epsilon}^{M}\left(\theta_{\epsilon}\right) \theta_{\epsilon} \\
& \leq \int_{\Omega} \hat{\gamma}_{\epsilon}\left(\theta_{\epsilon}(0)\right)+M \int_{\Sigma} f^{*} \leq C
\end{aligned}
$$

by (A5)-(i), the fact that $\widehat{\gamma}_{\epsilon} \geq 0$ is uniformly bounded in $[-M, M]$, the $L^{\infty}$ bound and (A5)-(iii).

The estimates (34) is standard; it suffices to take $\psi=p_{\epsilon}(t)$ in $(25)$.

Finally we obtain (33). Let $0<h<T$ be fixed and choose $0<t<T-h$. Take $\varphi=h^{-1}\left[\theta_{\epsilon}(t+h)-\theta_{\epsilon}(t)\right]$ in $(35)$ and integrate in $\tau \in(t, t+h)$, to get

$$
\begin{gathered}
\frac{\underline{b}}{h}\left\|\theta_{\epsilon}(t+h)-\theta_{\epsilon}(t)\right\|_{L^{2}(\Omega)}^{2} \\
\leq \frac{1}{h} \int_{\Omega}\left[\gamma_{\epsilon}\left(\theta_{\epsilon}(t+h)\right)-\gamma_{\epsilon}\left(\theta_{\epsilon}(t)\right)\right]\left[\theta_{\epsilon}(t+h)-\theta_{\epsilon}(t)\right] \\
=-\frac{1}{h} \int_{\Omega}\left(\int_{t}^{t+h} \nabla \theta_{\epsilon}(\tau) \mathrm{d} \tau\right) \cdot \nabla\left[\theta_{\epsilon}(t+h)-\theta_{\epsilon}(t)\right] \\
+\frac{1}{h} \int_{\Omega}\left(\int_{t}^{t+h}\left(d^{+}\left(\theta_{\epsilon}\right) \mathbf{w}_{\epsilon}^{*}\right)(\tau) \mathrm{d} \tau\right) \cdot \nabla\left[\theta_{\epsilon}(t+h)-\theta_{\epsilon}(t)\right] \\
-\frac{1}{h} \int_{\partial \Omega}\left(\int_{t}^{t+h}\left(f_{\epsilon}^{M}\left(\theta_{\epsilon}\right)\right)(\tau) \mathrm{d} \tau\right)\left[\theta_{\epsilon}(t+h)-\theta_{\epsilon}(t)\right]
\end{gathered}
$$


where the inequality is a consequence of (19). We now estimate the second term on the right hand side:

$$
\begin{gathered}
\frac{1}{h} \int_{\Omega}\left(\int_{t}^{t+h}\left(d^{+}\left(\theta_{\epsilon}\right) \mathbf{w}_{\epsilon}^{*}\right)(\tau) \mathrm{d} \tau\right) \cdot \nabla\left[\theta_{\epsilon}(t+h)-\theta_{\epsilon}(t)\right] \\
\leq \frac{1}{h} \max _{|s| \leq M}\left|d^{+}(s)\right|\left(h \int_{\Omega} \int_{t}^{t+h}\left|\mathbf{w}_{\epsilon}^{*}(\tau)\right|^{2} \mathrm{~d} \tau\right)^{\frac{1}{2}}\left(\int_{\Omega}\left|\nabla\left[\theta_{\epsilon}(t+h)-\theta_{\epsilon}(t)\right]\right|^{2}\right)^{\frac{1}{2}} \\
\leq \frac{1}{h} \max _{|s| \leq M}\left|d^{+}(s)\right|\left(h^{2} \sup _{t \leq \tau \leq t+h}\left\|\mathbf{w}_{\epsilon}^{*}(\tau)\right\|_{L^{2}(\Omega)}^{2}\right)^{\frac{1}{2}}\left\|\nabla\left[\theta_{\epsilon}(t+h)-\theta_{\epsilon}(t)\right]\right\|_{L^{2}(\Omega)} \\
\leq \max _{|s| \leq M}\left|d^{+}(s)\right|\left\|\mathbf{w}_{\epsilon}^{*}\right\|_{L^{\infty}\left(0, T ; L^{2}(\Omega)\right)}\left(\left\|\nabla \theta_{\epsilon}(t+h)\right\|_{L^{2}(\Omega)}+\left\|\nabla \theta_{\epsilon}(t)\right\|_{L^{2}(\Omega)}\right),
\end{gathered}
$$

using the inequality of Hölder, first in time and then in space, and the $L^{\infty}$ estimate. Concerning the third term we have, using a similar reasoning,

$$
\begin{gathered}
\quad-\frac{1}{h} \int_{\partial \Omega}\left(\int_{t}^{t+h}\left(f_{\epsilon}^{M}\left(\theta_{\epsilon}\right)\right)(\tau) \mathrm{d} \tau\right)\left[\theta_{\epsilon}(t+h)-\theta_{\epsilon}(t)\right] \\
\leq \frac{1}{h}\left(\int_{\partial \Omega} h \int_{t}^{t+h}\left|f_{\epsilon}^{M}\left(\theta_{\epsilon}\right)(\tau)\right|^{2} \mathrm{~d} \tau\right)^{\frac{1}{2}}\left(\int_{\partial \Omega}\left|\theta_{\epsilon}(t+h)-\theta_{\epsilon}(t)\right|^{2}\right)^{\frac{1}{2}} \\
\leq \frac{1}{h}\left(h^{2} \sup _{t \leq \tau \leq t+h}\left\|f_{\epsilon}^{M}\left(\theta_{\epsilon}\right)(\tau)\right\|_{L^{2}(\partial \Omega)}^{2}\right)^{\frac{1}{2}}\left\|\theta_{\epsilon}(t+h)-\theta_{\epsilon}(t)\right\|_{L^{2}(\partial \Omega)} \\
\leq\left\|f_{\epsilon}^{M}\left(\theta_{\epsilon}\right)\right\|_{L^{\infty}\left(0, T ; L^{2}(\partial \Omega)\right)}\left(\left\|\theta_{\epsilon}(t+h)\right\|_{L^{2}(\partial \Omega)}+\left\|\theta_{\epsilon}(t)\right\|_{L^{2}(\partial \Omega)}\right) \\
\leq\left\|f^{*}\right\|_{L^{\infty}(\Sigma)}\left(\left\|\theta_{\epsilon}(t+h)\right\|_{L^{2}(\partial \Omega)}+\left\|\theta_{\epsilon}(t)\right\|_{L^{2}(\partial \Omega)}\right)
\end{gathered}
$$

using (A5)-(iii).

To conclude, integrate the initial inequality in $t \in(0, T-h)$, obtaining

$$
\begin{gathered}
\int_{0}^{T-h} h^{-1}\left\|\theta_{\epsilon}(t+h)-\theta_{\epsilon}(t)\right\|_{L^{2}(\Omega)}^{2} \\
\leq \frac{1}{\underline{b}}\left\{\left\|\nabla \theta_{\epsilon}\right\|_{L^{2}(Q)}^{2}+2 \sqrt{T} \max _{|s| \leq M}\left|d^{+}(s)\right|\left\|\mathbf{w}_{\epsilon}^{*}\right\|_{L^{\infty}\left(0, T ; L^{2}(\Omega)\right)}\left\|\nabla \theta_{\epsilon}\right\|_{L^{2}(Q)}\right. \\
\left.+2 \sqrt{T}\left\|f^{*}\right\|_{L^{\infty}(\Sigma)}\left\|\theta_{\epsilon}\right\|_{L^{2}(\Sigma)}\right\} \equiv \kappa,
\end{gathered}
$$

because of the previous estimates and since

$$
\int_{0}^{T-h}-\frac{1}{h} \int_{\Omega}\left(\int_{t}^{t+h} \nabla \theta_{\epsilon}(\tau) \mathrm{d} \tau\right) \cdot \nabla\left[\theta_{\epsilon}(t+h)-\theta_{\epsilon}(t)\right]
$$




$$
\begin{aligned}
& =-\frac{1}{h} \int_{\Omega} \int_{0}^{T-h}\left(\int_{t}^{t+h} \nabla \theta_{\epsilon}(\tau) \mathrm{d} \tau\right) \frac{\mathrm{d}}{\mathrm{d} t}\left(\int_{t}^{t+h} \nabla \theta_{\epsilon}(\tau) \mathrm{d} \tau\right) \\
& =-\frac{1}{2 h} \int_{\Omega}\left\{\left(\int_{T-h}^{T} \nabla \theta_{\epsilon}(\tau) \mathrm{d} \tau\right)^{2}-\left(\int_{0}^{h} \nabla \theta_{\epsilon}(\tau) \mathrm{d} \tau\right)^{2}\right\} \\
& \leq \frac{1}{2 h} \int_{\Omega}\left(h \int_{T-h}^{T}\left|\nabla \theta_{\epsilon}\right|^{2}+h \int_{0}^{h}\left|\nabla \theta_{\epsilon}\right|^{2}\right) \leq\left\|\nabla \theta_{\epsilon}\right\|_{L^{2}(Q)}^{2}
\end{aligned}
$$

\section{Conclusion}

We have considered the analysis of a new generalized formulation for a phase change problem with convection arising in freezing and thawing of saturated porous media. By using the variational approach for this Darcy-Stefan problem, we were able to establish the existence of a generalized solution by introducing a suitable family of approximating problems that can, in principle, be numerically simulated by the finite element method. The solid/liquid interface is obtained a posteriori from the temperature field, without any requirement of regularity. This approach, based directly on the natural laws of conservation of mass and energy, is well adapted to rather general constitutive laws and has the advantage of being valid for general irregular geometries.

Acknowledgements. J.F. Rodrigues was partially supported by FCT, Praxis XXI and Praxis/2/2.1/MAT/125/94 and J.M. Urbano by CMUC/FCT, Praxis XXI and Praxis/2/2.1/MAT/458/94.

\section{References}

[1] Aguirre-Puente, J. and Frémond, M., Propagation du gel dans les milieux poreux, in "Application of Methods of Functional Analysis to Problems in Mechanics", Lecture Notes in Mathematics 503, Springer-Verlag, Berlin, 1976.

[2] Barret, J.W. and Elliott, C.M., A finite element method on a fixed mesh for the Stefan problem with convection in a saturated porous medium, in pp. 384409 of "Numerical Methods for Fluid Dynamics", K.W. Morton and M.J. Baines (Editors), Academic Press, 1982.

[3] Bear, J., "Dynamics of Fluids in Porous Media", American Elsevier, New York, 1972.

[4] Beckermann, C. and Viskanta, R., Natural convection solid/liquid phase change in porous media, Int. J. Heat Mass Transfer, 31 (1988), 35-46.

[5] Carey, G.F. (Editor), "Finite Element Modeling of Environmental Problems: Surface and Subsurface Flow and Transport", J. Wiley \& Sons, Chichester, 1995. 
[6] Chang, C.J. and Brown, R.A., Natural convection in steady solidification: finite element analysis of a two-phase Rayleigh-Bénard problem, J. Comput. Phys., 53 (1984), 1-27.

[7] Chipot, M. and Rodrigues, J.F., Comparison and stability of solutions to a class of quasilinear parabolic problems, Proceedings of the Royal Society of Edinburgh, 110A (1988), 275-285.

[8] DiBenedetto, E. and Elliott, C.M., Existence for a problem in ground freezing, Nonlinear Analysis, Th. Meth. Appl., 9 (1985), 953-967.

[9] DiBenedetto, E. and Friedman, A., Conduction-convection problems with change of phase, J. Diff. Eqs., 62 (1986), 129-185.

[10] DiBenedetto, E. and O'Leary, M., Three-dimensional conduction-convection problems with change of phase, Arch. Rational Mech. Anal., 123 (1993), 99-116.

[11] George, J.H., Gunn, R.D. and Straughan, B., Patterned ground formation and penetrative convection in porous media, Geophys. Astrophys. Fluid Dyn., 46 (1989), 135-158.

[12] Goldstein, M.E. and Reid, R.L., Effect of fluid flow on freezing and thawing of saturated porous media, Proc. Roy. Soc. London A, 364 (1978), 45-73.

[13] Kazmierczak, M. and Poulikakos, D., Melting of an ice surface in a porous medium, AIAA J. Thermophys. Heat Transfer, 2 (1988), 352-358.

[14] Ladyzenskaja, O., Solonnikov, V. and Ural'ceva, N., "Linear and Quasi-linear Equations of Parabolic Type", A.M.S. Transl. Monog. 23, Providence, 1968.

[15] McKay, G., Patterned ground formation and convection in porous media with a phase change, Continuum Mech. Thermodyn., 8 (1996), 189-199.

[16] Meirmanov, A.M., "The Stefan problem", De Gruyter, Berlin, 1992.

[17] Neittaanmäki, P. and Tiba, D., "Optimal Control of Nonlinear Parabolic Systems: Theory, Algorithms and Applications", M. Dekker, New York, 1994.

[18] Rodrigues, J.F., Variational Methods in the Stefan Problem, in pp. 147-212 of "Phase Tansitions and Hysteresis", A. Visintin (Editor), Lecture Notes in Mathematics 1584, Springer-Verlag, Berlin, 1994.

[19] Rodrigues, J.F., Steady state solutions to a multi-dimensional phase change problem in ground frezing, Proceedings of IUTAM Symposium "Variations of Domains and Free Boundaries", Paris, 1997.

[20] Rodrigues, J.F. and Urbano, J.M., On the stationary Boussinesq-Stefan problem with constitutive power-laws, Int. J. Non-Linear Mechanics, 33 (1998), 555-566.

[21] Rubinstein, L.I., "The Stefan problem", Amer. Math. Soc. Transl. Monogr. 27, Providence, 1971.

[22] Simon, J., Compact sets in the space $L^{p}(0, T ; B)$, Annali Mat. Pura ed Appl., 146 (1987), 65-96.

[23] Straughan, B., Mathematical aspects of penetrative convection, Pitman Res. Notes in Mathematics Series 288, Longman, Harlow, 1993.

[24] Zhang, X., Nguyen, T.H. and Kahawita, R., Melting of ice in a porous medium heated from below, Int. J. Heat Mass Transfer, 34 (1991), 389-405. 